\title{
INDICATIONS AND CONTRA-INDICATIONS IN RECONSTRUCTIVE SURGERY IN LEPROSY
}

\author{
by Johs. G. ANDERSEN, CAND. MED. ET CHIR. (HAFN.)
}

(Read at the Regional conference, Mission to Lepers, Purulia, January, 1963)

Reconstructive surgery has been with us for several years. It has passed the initial, experimental stage, and has arrived at a reasonably definite pattern that enables us to undertake a standard teaching and application of recognized patterns. It is - or should bea normal part of the service we offer to the sufferers from leprosy.

Scarcity of workers and lack of funds impose certain restrictions on the ideal conditions under which we would like to work.

What I have to say here, applies to a routine set up with competent workers that are not specifically engaged in teaching or research, that both have certain specific conditions outside the normal routine pattern of work.

The goal we should hold up for us is that every sufferer from leprosy with disabilities, amenable to surgery, should have the benefit of this work. This will not be possible for many years to come, maybe never. However, do not let us forget it.

There are certain general restrictions on the application of surgery that should be borne in mind. This will be particularly important where the selection of cases for surgery is not, or only partly, in the hands of a competent surgeon. The medical leprologist and the physiotherapist who may have to do the selection should keep these points in mind.

(1) Surgical treatment is only part of the general treatment of the patients. It must always be subordinate to the more important medical treatment, the aim of which is to enable the patient to return to society with an arrested or cured disease, offering no danger to his surroundings.

The general conditions are that the patient, chosen for surgery, should have been under regular, antileprosy treatment for not less than three months, and that he should not have presented any 'reaction' or signs of 'progressive disease' during that period, and that his therapy must be well stabilised before surgery.

(2) Grossly infected patients with a high bacillary index are unfit for surgery. The reason is the liability to reactions in these patients. I prefer to wait for the bacillary index to get below 1.0 before surgery. This does not mean that physiotherapy should be withheld from these patients. On the contrary, particularly in cases with violent reactions will certain types of preventive physiotherapy be of immense importance. 
(3) The pattern of paralysis should be stable. The majority of cases of paralysis in leprosy tend to develop toward and remain at certain, well recognized patterns of paralysis. Certain muscles are paralysed, and they are then completely paralysed. No other muscles are affected. That recovery of damaged or lost nerve function can take place, spontaneously or consequent to therapy is a known fact. It is too early to pass any opinion on the effect of the various possible means of therapy. It does seem to be a fact that in case a paralysis has remained complete and stable for 8 weeks no possibility of recovery can be expected. No attempt at reconstructive surgery is indicated in this interval.

The surgeon or physiotherapist, trained in reconstructive surgery in leprosy is trained to detect and evaluate these patterns. It is necessary for the leprologist with no such training to consult either a physiotherapist or a surgeon in this matter. Disregard of this problem can be quite disastrous, since the results of surgery tend to become less impressive the more frequently operations are undertaken, and also because the technique may be quite different in a case with extensive paralysis from a case with limited paralysis. The less extensive surgery may make the more extensive surgery very difficult, certainly less satisfactory.

(4) No patient in poor general health is a good surgical risk. Proper care should be taken in this respect, so that we may offer the patient the best chances of a good result.

(5) Since reconstructive surgery almost exclusively deals with tendons, bones, joints and valuable skin, postoperative infections have a most distressing result. More than in any other branch of surgery we work under the maxim of 'once done, never redone'. The surgeon himself must be on his guard. He will naturally be mostly concerned with the influence of his technique on the risk of infection. But the condition of the skin in the involved area and generally should be of prime importance, also to the selecting medical officer. Skin rashes and particularly scabies can have horrible effects and are easily overlooked.

In the same category fall allergic conditions of the skin.

(6) Concomitant diseases, bowel diseases, upper respiratory infections, etc., are always troublesome and can be damaging to the final result.

(7) A w'ord about antibiotics. Enough care cannot be executed in the use of these extremely valuable drugs. Some of us will recall the hopeful optimism about surgery under an 'antibiotic umbrella'. We know better now. Actually an antibiotic umbrella rather promotes infection with resistant and more dangerous strains. The highly unpleasant, not infrequently life-threatening side effects should make us extremely wary. Particularly in the treatment of plantar ulcers are many likely to shorten the period of treatment and 
give 'just a few shots of penicillin' or whichever drug holds our fancy at present. It may conceivably have a good effect, occasionally, but it is a dangerous attitude. Good clean surgical procedures, as they have been laid down again and again over centuries are far better. And they are cheaper. Nobody has described or are likely to detect resistance to soap bath or mercurochrome. Nor can any antibiotic replace the physiological effect of immobilisation in a plaster of Paris cast or a Karigiri boot, properly applied.

We are, however, not only concerned with a medical aspect of our patients. Of equal importance is the fact that they should return to as closely as possible a normal life in a normal society-and as quickly as possible. It is an unpleasant, but unavoidable fact that certain persons are more likely to return to a normal society than others. We have a responsibility toward the individual man. But we also have a responsibility towards the society in which we live. That indicates certain preferences for our work.

Under otherwise equal conditions I would suggest that we give preference to young people, to people who actually hold a job, and to people with a responsible attitude to life.

Without going into details I would like to point out a few important considerations, relative to the selection of cases for particular kinds of reconstructive surgery.

(1) Intra-ocular surgery in leprosy is a most difficult and tricky discipline. I would strongly advise anybody who does not have considerable experience in opthalmology and leprosy not to undertake it. Precautions against reactions apply here with manifold force. A firmly negative bacillary index and a perfectly stationary morbid condition are obligatory.

(2) Temporalis transfer a.m. Gillies, the accepted method for correction of paralytic lagohthaloms, is an excellent prevention of the dreaded blindness. In experienced hands satisfactory results can be expected in close to $100 \%$ of the cases. Prevention of blindness is so urgent that nobody should be excluded, not even the confirmed beggar who defies all attempts at rehabilitation. The rules governing bacillary index may be slackened considerably. The only really valid contraindications are frank reaction and serious infection of the face. This is probably the only condition where it can be advisable to operate under the dangerous 'corticosteroid umbrella'.

(3) Surgery on bone in leprosy is concerned both with the effect of anaesthesia/infection and with direct leproma infiltration into bone. Together with surgery of the nerve this is the least explored and understood part of the discipline. The conditions relating to the morbid condition and to possibilities of secondary infection should be strictly observed.

(4) Ulcer surgery of ten deals with bone surgery, but is primarily a means to secure safe healing of the weightbearing surfaces. It 
requires careful and expert evaluation of tissue, and even in competent hands the results are not very encouraging. The happy chopping away of diseased bone that was, and I believe still is, an important part of the ulcer regime in many places, should be discarded. Ulcer surgery is essentially a losing battle, where every millimetre of bone counts. Factors specific to leprosy make it extremely difficult, even for experienced radiologists and orthopaedic surgeons, to judge the viability of involved bone. So be careful and play it safe. Before actual surgery is resorted to the good old conservative methods of immobilization and drainage of frank infections should be given a very generous trial.

(5) The conditions grouped round the paralytic drop foot are probably the most rewarding for the surgeon. Given good skin conditions and a reasonably clear field as far as leprosy is concerned the only worry is really the presence of frank or obscured ulcers with deep seated smouldering infection of the foot. The freely mobile, but stable foot with no angular deviations or posterior contractures is naturally the choice for a perfect result, but even much worse feet can yield quite satisfactory results.

(6) In surgery of the hand conditions that are likely to yield poor results should be recognized. Extensive paralysis, particularly involving the radial nerve, always gives a comparatively poor result. The same is true of the long standing paralysis with stiff joints that do not respond to physiotherapy. The effects of anaesthesia/infection, particularly on the joints also tend to give less satisfactory results.

The problems concerned with the selection of cases for hand surgery throw light over a very important consideration. It may be possible to obtain a better result if you subject the patient to prolonged periods of physiotherapy. Are we justified in aiming at a near perfect result if this means so long absence of the patient from his normal surroundings that he comes home a social wreck with a near perfect surgical result? After all the social rehabilitation of the patient is the final test of our therapy.

(7) Surgery of the face, notably the nose and the eyebrows, is more than any other branch of the discipline a direct attempt at social rehabilitation. We who are accustomed to being with these deformed patients may not consider madarosis of the eyebrows or a collapsed nose a serious disability compared with a claw hand or a drop foot. To the person looking for a job in a normal society it may quite easily be the cornerstone of his surgical reconstruction. After all, what is the good of being able to do a job, if nobody wants to employ you?

The structures we deal with render a strict observation of the rules governing the morbid conditions extremely important.

I would like to add a few suggestions for the planning of this service. We should recognize that it calls for highly trained, dedicated 
people. I do not want to be unduly pessimistic, but we are not likely to be flooded with trained physiotherapists or surgeons. And the job certainly is overwhelmingly big. This should compel us to think in rational lines about the way in which we can utilise available personnel and funds. I should imagine that the logical way would be to build up good centres at suitable places and then extend a real co-operation with outlying colonies and hospitals. 